\title{
Noise and Correlations in a Spatial Population Model with Cyclic Competition
}

\author{
Tobias Reichenbach, Mauro Mobilia, ${ }^{*}$ and Erwin Frey \\ Arnold Sommerfeld Center for Theoretical Physics (ASC) and Center for NanoScience (CeNS), Department of Physics, \\ Ludwig-Maximilians-Universität München, Theresienstrasse 37, D-80333 München, Germany
}

(Received 19 July 2007; published 7 December 2007)

\begin{abstract}
Noise and spatial degrees of freedom characterize most ecosystems. Some aspects of their influence on the coevolution of populations with cyclic interspecies competition have been demonstrated in recent experiments [e.g., B. Kerr et al., Nature (London) 418, 171 (2002)]. To reach a better theoretical understanding of these phenomena, we consider a paradigmatic spatial model where three species exhibit cyclic dominance. Using an individual-based description, as well as stochastic partial differential and deterministic reaction-diffusion equations, we account for stochastic fluctuations and spatial diffusion at different levels and show how fascinating patterns of entangled spirals emerge. We rationalize our analysis by computing the spatiotemporal correlation functions and provide analytical expressions for the front velocity and the wavelength of the propagating spiral waves.
\end{abstract}

DOI: $10.1103 /$ PhysRevLett.99.238105

Understanding the combined influence of spatial degrees of freedom and noise on biodiversity is an important issue in theoretical biology and ecology. This implies facing the challenging problem of studying complex nonequilibrium structures, which form in the course of nonlinear evolution [1-6]. More generally, self-organized nonequilibrium patterns and traveling waves are ubiquitous in nature and appear, for instance, in chemical reactions, biological systems, as well as in epidemic outbreaks [7]. Among the most studied types of patterns are spiral waves, which are relevant to autocatalytic chemical reactions, aggregating slime-mold cells, and cardiac muscle tissue [8]. In all these nonequilibrium and nonlinear processes, as well as in population dynamics models [1,3,5], pattern formation is driven by diffusion which, together with internal noise, act as mechanisms allowing for stabilization and coevolution of the reactants. In this work, we consider a paradigmatic spatially extended 3 species population system with cyclic competition, which can be regarded as a simple food-chain model [9]. In fact, such a system is inspired by recent experiments on the coevolution of 3 species of bacteria in cyclic competition [4]. Using methods of statistical physics, we study the influence of spatial degrees of freedom and internal noise on the coevolution of the species and on the emerging spiral patterns. In particular, we compute the correlation functions and provide analytical expressions for the spreading speed and wavelength of the propagating fronts. To underpin the role of internal noise, the results of the stochastic description are compared with those of the deterministic equations.

In this Letter, we investigate a stochastic spatial variant of the rock-paper-scissors game [9] (also referred to as cyclic Lotka-Volterra model). These kinds of systems have been studied both from a game-theoretic perspective, see, e.g., $[10,11]$ and references therein, and within the framework of chemical reactions [5,12], revealing rich spatiotemporal behaviors (e.g., emergence of rotating spirals). While our methods have a broad range of applicability,
PACS numbers: 87.23.Cc, 02.50.Ey, 05.10.Gg, 87.18.Hf

they are illustrated for a prototypical model introduced by May and Leonard [13] where 3 species, $A, B$, and $C$, undergo a cyclic competition (codominance with rate $\sigma$ ) and reproduction (with rate $\mu$ ), according to the reactions

$$
\begin{array}{ccc}
A B \stackrel{\sigma}{\rightarrow} A \oslash, & B C \stackrel{\sigma}{\rightarrow} B \oslash, & C A \stackrel{\sigma}{\rightarrow} C \oslash, \\
A \oslash \stackrel{\mu}{\rightarrow} A A, & B \oslash \stackrel{\mu}{\rightarrow} B B, & C \oslash \stackrel{\mu}{\rightarrow} C C .
\end{array}
$$

Hence, an individual of species $A$ will consume one of species $B(A B \rightarrow A \oslash)$ with rate $\sigma$ and will reproduce with rate $\mu$ if an empty spot, denoted $\oslash$, is available $(A \oslash \rightarrow A A$, i.e., there is a finite carrying capacity). In addition, to mimic the possibility of migration, it is realistic to endow the individuals with a form of mobility. For the sake of simplicity, we consider a simple exchange process, with rate $\epsilon$, among any nearest-neighbor pairs of agents: $X Y \stackrel{\epsilon}{\rightarrow} Y X$, where $X, Y \in\{A, B, C, \oslash\}$. If one ignores the spatial structure and assumes the system to be well mixed (with an infinite number of individuals), the population's mobility plays no role and the dynamics is aptly described by the deterministic rate equations (RE) for the densities $a$, $b, c$ of species $A, B$, and $C$, respectively. Introducing $s \equiv$ $(a, b, c)$, the RE read:

$$
\partial_{t} s_{i}=s_{i}\left[\mu(1-\rho)-\sigma s_{i+2}\right], \quad i \in\{1,2,3\},
$$

where the index $i$ is taken modulo 3 and $\rho=a+b+c$ is the total density. As shown by May and Leonard [13] (see also [14]), these equations possess 4 absorbing fixed points, corresponding to a system filled with only one species and to an empty system. In addition, there is a reactive fixed point $\boldsymbol{s}^{*}=\frac{\mu}{\sigma+3 \mu}(1,1,1)$, corresponding to a total density $\rho^{*}=\frac{3 \mu}{\sigma+3 \mu}$. A linear stability analysis shows that $s^{*}$ is unstable. The absorbing steady states $(1,0,0),(0$, $1,0)$, and $(0,0,1)$ are heteroclinic points. The existence of a Lyapunov function $\mathcal{L}=a b c / \rho^{3}$ allows us to prove that, within the realm of the above RE, the phase portrait is characterized by flows spiraling outward from $s^{*}$, with 
frequency $\omega_{0}=\sqrt{3} \mu \sigma /[2(3 \mu+2 \sigma)]$ in its vicinity. Approaching the boundaries of the phase portrait, the trajectories form (heteroclinic) cycles indefinitely close to the edges (without ever reaching them), with densities approaching, in turn, the value one. Despite its mathematical elegance, this behavior has been recognized to be unrealistic [13,14]. In fact, for finite populations, fluctuations arise and always cause the extinction of two species in finite time (see, e.g., Ref. [15]).

In this work, considering the spatial version of the above model in the presence of internal noise, we show that a robust (and, arguably, more realistic) scenario for the evolution arises. The reaction schemes (2) and the exchange events are considered to occur on a $d$-dimensional regular lattice of $N$ sites, labeled $\boldsymbol{r}=$ $\left(r_{1}, \ldots, r_{d}\right)$. Each lattice site has $z$ neighbors at a distance $\delta r$ (e.g., $z=2 d$ and $N=L^{d}$ for hypercubic lattices of linear size $L$ ) and is either empty or occupied by at most one individual. On the lattice, the binary reactions (1) and exchanges only occur among pairs of nearest neighbors. In the situation of large system sizes, the continuum limit reveals that for the exchange process to be an efficient driving mechanism, the rate $\epsilon$ has to scale as $\epsilon \propto N^{\nu}$, with $\nu=2 / d$ and $N \rightarrow \infty$. In fact, if $0<\nu<2 / d$ the system is dominated by the local reactions (1) among neighboring individuals, while effective diffusion renders locality irrelevant when $\nu>2 / d$. Only when $\nu=2 / d$ is there an effective competition between the stirring process and the local reactions (1). It is therefore useful to introduce the effective diffusion constant $D \equiv \frac{z}{2 d^{2}} N^{-2 / d} \epsilon$. Because of the discreteness of the number of individuals involved in the reactions, internal fluctuations arise in the system. The latter originate from (i) the interspecies reactions (1) and (ii) the exchange processes. In the continuum limit, where $\delta r=N^{-1 / d}$ with $N, \epsilon \rightarrow \infty$ (and finite $D$ ), there is a separation of time scales and the pair exchanges occur much faster than the reactions $\left(\epsilon \propto N^{2 / d}\right)$. Actually, the fluctuations associated with (1) and the agents' mobility scale, respectively, as $N^{-1 / 2}$ and $N^{-1}$, with the former dominating over the latter and being the only relevant contribution. This result is revealed by a system size, also called Kramers-Moyal (see, e.g., Ref. [16], Chap. 8), expansion (SZE) of the master equation underlying the exchange processes and the reactions (1) [17]. Furthermore, the SZE yields a proper Fokker-Planck equation, which is equivalent to a set of (Ito) stochastic partial differential equations (SPDE) with white noise. The derivation, obtained in the continuum limit from the master equation, is outlined in the supplementary EPAPS document [17] and will be detailed elsewhere [18]. Here, we quote the expression of the SPDE:

$$
\partial_{t} s_{i}=D \nabla^{2} s_{i}+\mathcal{A}_{i}(s)+\sum_{j=1}^{3} \mathcal{C}_{i j}(s) \xi_{j}, \quad i \in\{1,2,3\},
$$

where $\nabla^{2}$ is the Laplacian operator; $\left\langle\xi_{i}(\boldsymbol{r}, t)\right\rangle=0$,

$$
\begin{gathered}
\left\langle\xi_{i}(\boldsymbol{r}, t) \xi_{j}\left(\boldsymbol{r}^{\prime}, t^{\prime}\right)\right\rangle=\delta_{i, j} \delta\left(\boldsymbol{r}-\boldsymbol{r}^{\prime}\right) \delta\left(t-t^{\prime}\right), \text { and } \\
\mathcal{A}_{i}(s)=s_{i}\left[\mu(1-\rho)-\sigma s_{i+2}\right], \\
\mathcal{C}_{i j}(s)=\delta_{i j} \sqrt{N^{-1} s_{i}\left[\mu(1-\rho)+\sigma s_{i+2}\right]} .
\end{gathered}
$$

Again, the indices are taken modulo 3 and now $s_{i} \equiv$ $s_{i}(\boldsymbol{r}, t)$. As explained in [16,18], these SPDE have to be interpreted in the sense of Ito calculus. While Eqs. (3) and our approach are valid in any dimension [17], for specificity, we now analyze the spatiotemporal properties of the system in two dimensions with periodic boundary conditions. On the one hand we have solved numerically the SPDE (3) using the open software from the XMDS project [19]. On the other hand, we have carried out individualbased simulations of the reactions (1) for mobile (exchange process) particles on lattices of size $L \times L$, with $L=$ 30-1000. This allows to carefully study the convergence towards the continuum limit, where the description in terms of (3) is expected to be accurate.

As other spatially extended dynamical systems [5,1012], the model under consideration displays fascinating nonequilibrium patterns emerging in the course of the evolution. In Fig. 1(a) and 1(b), we report typical longtime snapshots of the system for low (a) and high (b) exchange rates (but keeping $D$ fixed), as obtained from lattice simulations. In both cases we notice that intriguing patterns form. For slow exchange rate, the system displays nongeometrical patches, similarly to what happens in systems with self-organized criticality [20]. When the exchange rate is raised, the patterns display spiral structures. In fact, starting from a spatially homogeneous initial condition, $s(r, 0)=s^{*}$, the system is randomly perturbed by the internal noise and the resulting spatial inhomogeneities grow and form wave fronts moving through the system. The emergence of spiral patterns is a feature shared by other excitable systems (see, e.g., [7,8]) and corresponds to the ability of the system to sustain the propagation of oscillating waves. For sufficiently large $\epsilon$, one observes a striking resemblance between the size and

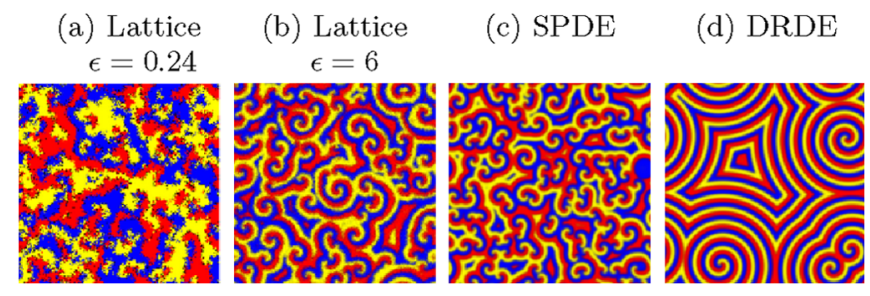

FIG. 1 (color online). Snapshots of reactive steady states for rates $D=3 \times 10^{-6}, \mu=\sigma=1$. Each color (level of gray) indicates one species (black dots correspond to vacancies). In (a) and (b) results are from lattice simulations for $L=200$ (a) and $L=1000$ (b), i.e., different $\epsilon$. Spiral structures emerge for sufficiently large exchange rate (b). Numerical solution of the SPDE (3) and DRDE are shown in (c), respectively (d); see text. In (a)-(c), initially $s(r, 0)=s^{*}$. 
structure of the patterns obtained from the lattice simulations [Fig. 1(b)] and those from the SPDE (3) [Fig. 1(c)]. To further compare the predictions of the SPDE (3) with the lattice simulations, and to gain additional information on the structure of the emerging patterns, we have computed the correlation functions, $g_{s_{i} s_{j}}\left(\boldsymbol{r}-\boldsymbol{r}^{\prime}, t\right) \equiv$ $\left\langle s_{i}(\boldsymbol{r}, t) s_{j}\left(\boldsymbol{r}^{\prime}, t\right)\right\rangle-\left\langle s_{i}(\boldsymbol{r}, t)\right\rangle\left\langle s_{j}\left(\boldsymbol{r}^{\prime}, t\right)\right\rangle$ in two dimensions. In Fig. 2 (red and blue curves), we report the results for $g_{a a}(\boldsymbol{r}, t)$ in the steady state and notice an excellent agreement between the results of the lattice simulations and the predictions of the SPDE (3). The inset of Fig. 2 displays the correlation length $\ell_{\text {corr }}$ [21] as a function of $\epsilon(D$ is kept fixed, $L$ varies) obtained in the lattice simulations, which is found to coincide with the prediction of the SPDE already for $\epsilon \geq 5$. We have also computed the autocorrelation function $g_{s_{i} s_{j}}(0, t)$ and found, both in the lattice simulations and from the solutions of the SPDE, an oscillating behavior with a similar characteristic frequency, markedly different from $\omega_{0}$ [18]. This confirms that, even for finite exchange rates, the solution of the SPDE (3) provides an excellent approximation of the lattice simulations of the system. This is rather surprising since Eqs. (3) have been derived in the continuum limit, where $N$ and $\epsilon \rightarrow \infty$. A comparable influence of finite exchange rate in a predator-prey system has been reported recently [22]. According to the SPDE (3), $\ell_{\text {corr }}$ scales as $D^{1 / 2}$, so that by raising the diffusion one increases the size of the spirals. As we have shown in Ref. [6], this happens up to a critical value $D_{c}$ (e.g., $D_{c} \approx$ $4.5 \pm 0.5 \times 10^{-4}$ for $\mu=\sigma=1$ ): above that threshold, the spiral structures outgrow the system size and only one species survives, corresponding to an absorbing steady state predicted by Eqs. (2).

As the properties of the lattice simulations are well captured by the SPDE (3), where the strength of the noise

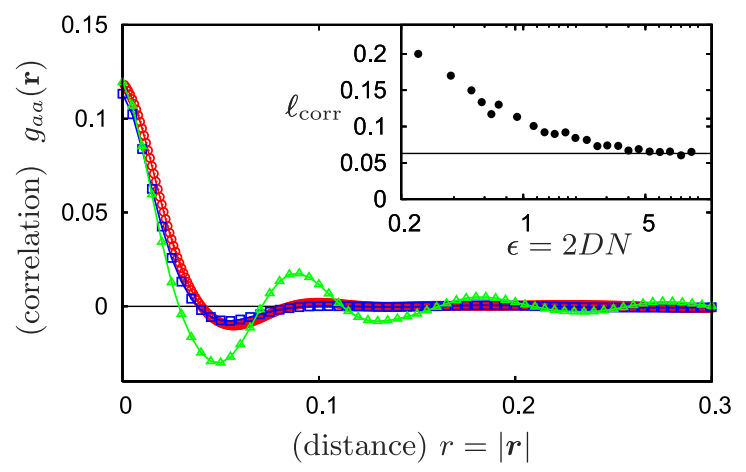

FIG. 2 (color online). Spatial correlation functions in 2D, obtained from lattice simulations (red, circles; $\epsilon=6, L=$ 1000), from the solution of the SPDE (3) (dark blue, squares) and of the DRDE (green, triangles), see text. The reaction rates are $\mu=\sigma=1$ and $D=3 \times 10^{-6}$. Inset: the correlation length $\ell_{\text {corr }}$, for $D=5 \times 10^{-5}, \mu=\sigma=1$, as function of $\epsilon$ (i.e., for different lattice sizes) compared to the prediction of the SPDE (black line). The latter is in excellent agreement with lattice simulations already for $\epsilon \geq 5$ (i.e., $L \geq 225$ ). scales as $N^{-1 / 2}$, with $N \rightarrow \infty$, it is natural to investigate the actual influence of this internal noise on the steady state of the system. To address this issue, we have solved numerically (in 2D, with periodic boundary conditions) the deterministic reaction-diffusion equation (DRDE) obtained from (3) by dropping the noise terms, i.e., $\partial_{t} s_{i}=D \nabla^{2} s_{i}+$ $\mathcal{A}_{i}(s)$. Of course, to obtain a nontrivial steady state for the DRDE one has to assume spatially inhomogeneous initial conditions. In Fig. 1(d), we have reported a snapshot of the long-time behavior predicted by the DRDE starting from $\boldsymbol{s}(\boldsymbol{r}, 0)=\boldsymbol{s}^{*}+\left(\frac{1}{100} \cos 2 \pi r_{1} r_{2}, 0,0\right)$. In this case, the dynamics evolves towards a reactive steady state which also exhibits spiral waves. However, the latter do not form entangled structures, but ordered geometrical patterns. As an example, only four spirals cover the system in Fig. 1(d) [while noise leads to 106 entangled spirals in Fig. 1(c)]. The correlation functions associated with the DRDE therefore exhibit only weakly damped spatial oscillations (see Fig. 2, green triangles). By analyzing typical snapshots like those of Fig. 1(d), we have noted that in the deterministic and stochastic [i.e., lattice simulations with "large" $\epsilon$ and solutions of Eqs. (3)] descriptions, the spiral waves share the same propagation velocity, frequency, and wavelength. However, a major difference between these descriptions lies on the crucial dependence of the DRDE on initial conditions, which determine the overall number of spirals and their size. On the contrary, because the internal noise acts a random source of spatial inhomogeneities, the lattice stochastic system and the SPDE display robust features. In particular, we have found noise to induce a universal spiral density of about 0.5 per square wavelength.

Analytical expressions for the spreading velocity and the wavelength of the propagating fronts of the DRDE can be obtained by considering the dynamics on the invariant manifold of the RE [23], given by $\mathcal{M}:\left\{y_{C}=\frac{\sigma(3 \mu+\sigma)}{4 \mu(3 \mu+2 \sigma)} \times\right.$ $\left.\left(y_{A}^{2}+y_{B}^{2}\right)+O\left(y^{3}\right)\right\}$, with

$$
\left(y_{A}, y_{B}, y_{C}\right)^{T} \equiv \frac{1}{3}\left(\begin{array}{ccc}
\sqrt{3} & 0 & -\sqrt{3} \\
-1 & 2 & -1 \\
1 & 1 & 1
\end{array}\right)\left(s^{T}-s^{* T}\right) .
$$

On $\mathcal{M}$, up to 3 rd order, the DRDE can be recast in the form of a forced complex Ginzburg-Landau equation (CGLE) $[24,25]$. By performing the nonlinear transformation $z_{A}=y_{A}+\frac{3 \mu+\sigma}{28 \mu}\left[\sqrt{3} y_{A}^{2}+10 y_{A} y_{B}-\sqrt{3} y_{B}^{2}\right]$ and $z_{B}=$ $y_{B}+\frac{3 \mu+\sigma}{28 \mu}\left[5 y_{A}^{2}+2 \sqrt{3} y_{A} y_{B}-5 y_{B}^{2}\right]$, upon ignoring nonlinearities like $\left(\nabla z_{A, B}\right)^{2}$, one is left with the following CGLE in the variable $z \equiv z_{A}+i z_{B}[18]$ :

$$
\partial_{t} z=D \nabla^{2} z+\left(c_{1}-i \omega_{0}\right) z-c_{2}\left(1+i c_{3}\right)|z|^{2} z,
$$

with $\quad c_{1} \equiv \frac{\mu \sigma}{2(3 \mu+\sigma)}, \quad c_{2} \equiv \frac{\sigma(3 \mu+\sigma)(48 \mu+11 \sigma)}{56 \mu(3 \mu+2 \sigma)}, \quad$ and $\quad c_{3} \equiv$ $\frac{\sqrt{3}(18 \mu+5 \sigma)}{(48 \mu+11 \sigma)}$. The general theory of front propagation [24,25] predicts that Eq. (6) always admits traveling waves as stable solutions (i.e., no Benjamin-Feir or Eckhaus instabilities occur). We have determined such periodic 


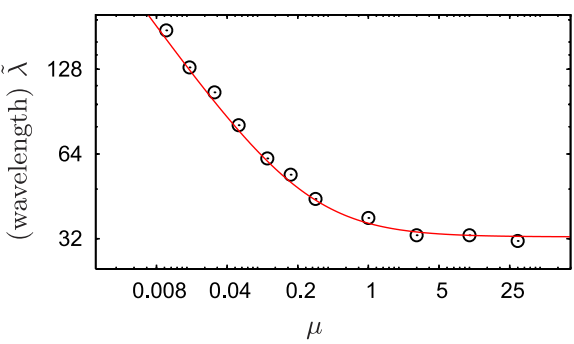

FIG. 3 (color online). Plot of $\tilde{\lambda}$, where $\lambda=\tilde{\lambda} \sqrt{D}$ is the spirals' wavelength of the propagating spiral waves. Analytical results (red curve, rescaled by a factor 1.6; see text) are compared with the solution of the SPDE (3) (black circles).

solutions by computing, from the dispersion relation of (6), the spreading velocity $v$ and the spirals' wavelength $\lambda$ (details will be given in [18]):

$$
v=2 \sqrt{c_{1} D}, \quad \lambda=2 \pi c_{3} \sqrt{c_{1}^{-1} D}\left(1-\sqrt{1+c_{3}^{2}}\right)^{-1} .
$$

In the stochastic version of the model, the wavelength and velocity of the wave fronts have been found to agree with those of the deterministic treatment. Hence, the expressions (7) also apply (for large $\epsilon$, with $D<D_{c}$ ) to the results of lattice simulations (rescaled by a factor $L$ ) and to the solution of the SPDE (3). For instance, on a square grid with $\mu=\sigma=1$, lattice simulations and Eqs. (3) yield $v \approx 0.63 D^{1 / 2} L$, in good agreement with the prediction of (7): $v=(D / 2)^{1 / 2} L$. For the spirals' wavelength, numerical results (lattice simulations and SPDE) yield $\lambda \propto D^{1 / 2}$ as predicted by (7). In Fig. 3, the analytical prediction (7) for $\lambda$ is compared with the values obtained from the SPDE (3), yielding a remarkable agreement for the functional dependence on the parameter $\mu$. Yet, as Eq. (6) does not account for all nonlinearities, the analytical and numerical values differ by a prefactor $\approx 1.6$ (considered in Fig. 3 ) [18]. It can still be noted that (6) and the predictions (7) are valid in all dimensions $[17,18]$.

Motivated by recent experiments [4], we have considered a spatially extended model with three species in cyclic competition and focused on the spatial and stochastic effects. The local character of the reactions and internal noise allow mobile populations to coexist and lead to pattern formation. We have shown that already for finite mobility the lattice model can be described by SPDE. With the latter and lattice simulations, we have studied how entanglement of spirals form and we have obtained expressions for their spreading velocity and wavelength. The size of the patterns crucially depends on the diffusivity: above a certain threshold the system is covered by one species [6]. In the absence of noise, the equations still predict the formation of spiral waves, but their spatial arrangement depends on the initial conditions.

Support of the German Excellence Initiative via the program "Nanosystems Initiative Munich" and of the Grant No. SFB TR 12 are gratefully acknowledged.
M. M. is grateful to the Alexander von Humboldt Foundation for support through the Grant No. IV-SCZ/1119205.

*Current address: Mathematics Institute \& Complexity Complex, The University of Warwick, Coventry CV4 7AL, United Kingdom.

[1] A. M. Turing, Phil. Trans. R. Soc. B 237, 37 (1952).

[2] R. M. May, Stability and Complexity in Model Ecosystems (Cambridge University Press, Cambridge, England, 1974); S. J. Maynard, Models in Ecology (Cambridge University Press, Cambridge, England, 1974).

[3] J.D. Murray, Mathematical Biology (Springer-Verlag, Berlin, 2002).

[4] B. Kerr et al., Nature (London) 418, 171 (2002).

[5] K. I. Tainaka, Phys. Rev. Lett. 63, 2688 (1989); Phys. Rev. E 50, 3401 (1994).

[6] T. Reichenbach, M. Mobilia, and E. Frey, Nature (London) 448, 1046 (2007).

[7] Chemical Waves and Patterns, Part One, edited by R. Kapral and K. Showalter (Kluwer Academic Publishers, Dordrecht, 1995); J. Lechleiter et al., Science 252, 123 (1991); B. T. Grenfell, O. N. Bjornstad, and J. Kappey, Nature (London) 414, 716 (2001).

[8] A. N. Zaikin and A.M. Zhabotinskii, Nature (London) 225, 535 (1970).

[9] J. Hofbauer and K. Sigmund, Evolutionary Games and Population Dynamics (Cambridge University Press, Cambridge, England, 1998).

[10] C. Hauert and G. Szabó, Am. J. Phys. 73, 405 (2005).

[11] G. Szabó and G. Fáth, Phys. Rep. 446, 97 (2007).

[12] L. Frachebourg, P. L. Krapivsky, and E. Ben-Naim, Phys. Rev. Lett. 77, 2125 (1996).

[13] R. M. May and W. J. Leonard, SIAM J. Appl. Math. 29, 243 (1975).

[14] R. Durrett and S. Levin, Theor. Popul. Biol. 53, 30 (1998).

[15] T. Reichenbach, M. Mobilia, and E. Frey, Phys. Rev. E 74, 051907 (2006).

[16] C. W. Gardiner, Handbook of Stochastic Methods (Springer, New York, 1983), 1st ed.

[17] See EPAPS Document No. E-PRLTAO-99-046747 for two appendices. In Appendix A, we outline the derivation of the stochastic partial differential equations (3) from the underlying master equation. Appendix B is devoted to a brief and general discussion of the properties of the system in arbitrary dimensions. For more information on EPAPS, see http://www.aip.org/pubservs/epaps.html.

[18] T. Reichenbach, M. Mobilia, and E. Frey (to be published).

[19] http://www.xmds.org.

[20] K. Schenk et al., Eur. Phys. J. B 15, 177 (2000).

[21] The correlation length denotes the characteristic length at which the spatial correlations decay by a factor $1 / e$ from their maximal value.

[22] M. Mobilia, I. T. Georgiev, and U. C. Täuber, Phys. Rev. E 73, 040903(R) (2006).

[23] S. Wiggins, Introduction to Applied Nonlinear Dynamical Systems and Chaos (Springer, New York, 1990), 1st ed.

[24] W. Saarloos, Phys. Rep. 386, 29 (2003).

[25] M. C. Cross and P. C. Hohenberg, Rev. Mod. Phys. 65, 851 (1993). 